Ciência e Natura, Santa Maria v.38 Ed. Especial-

IX Workshop Brasileiro de Micrometeorologia, 2016, p. 367 - 371

Revista do Centro de Ciências Naturais e Exatas - UFSM

ISSN impressa: 0100-8307 ISSN on-line: 2179-460X

\title{
CIÊNCIAPNATURA 35兽
}

\section{Calibração de equações Clássicas para radiação de onda longa na Região de Pampa em dias de céu claro}

Classic calibration equations for long wave radiation in the Pampa region on a clear day

\section{Daniele Morgenstern. Aimii ${ }^{*}$, Débora Regina Roberti ${ }^{1}$; Silvana Maldaner2; Gervazio Degrasia ${ }^{1}$; Marcelo Diaz ${ }^{1}$; Edyson Waghetti Sebastiany ${ }^{1}$ e Gisele Cristina Rubert ${ }^{1}$}

\author{
${ }^{1}$ Departamento de Física/Laboratório de Micrometeorologia, UFSM, Santa Maria, RS, Brasil. \\ danielefm@gmail.com; debora@ufsm.br; gervasiodegrasia@gmail.com; marbdiaz@gmail.com; \\ surycktheone@hotmail.com; girubert@gmail.com; \\ ${ }^{2}$ Coordenadoria acadêmica - UFSM Campus de Cachoeria do Sul \\ silvana.maldaner@gmail.com
}

\begin{abstract}
Resumo
A radiação de onda longa atmosférica é uma quantidade fundamental no cálculo do balanço de radiação na superfície. Medidas destc grandeza na região do Pampa gaucho são escassas. Uma alternativa para estimar esta variável é o emprego de equações clássicas que utilizam valores de temperatura do ar e da pressão de vapor. O objetivo deste estudo foi avaliar o desempenho de cinco equações na estimativa da radiação de onda longa atmosférica para dias de céu claro em uma área bioma Pampa localizada na cidade de Santa Maria-RS. Medidas de radiação de onda longa atmosférica realizadas durante todo o ano de 2014 no sítio de Santa Maria/Brasil foram utilizadas para validar c desempenho das equações. Após as estimativas de radiação pelas equações clássicas, um ajuste por mínimos quadrados foi empegando par calibrar tais equações ao bioma do Pampa. Com o ajuste observou-se que a equação de Brutsaert (1975) foi a que melhor descreveu a radiaçã de onda longa obsevada na região do PAMPA.
\end{abstract}

Palavras-chave: Radiação de onda longa, Pampa, céu claro, temperatura do ar, pressão de vapor.

\begin{abstract}
The atmospheric longwave radiation is a fundamental quantity in calculating the radiation balance at the surface. Measures of this magnitude in the gaucho Pampas region are scarce. An alternative estimate for this variable is the use of classical equations that use ai temperatures and steam pressure. The objective of this study was to evaluate the performance of five equations in the estimation of the lone atmospheric wave radiation for a clear day on a Pampa biome located in Santa Maria-RS. Atmospheric longwave radiation measurements taken throughout the year 2014 on the site of Santa Maria / Brazil were used to validate the performance of equations. After the radiatior estimates by classical equations, an adjustment by least squares was empegando to calibrate such equations to the biome Pampa. By adjusting it noted that the equation Brutsaert (1975) was best described obsevada long wave radiation in the PAMPA region.
\end{abstract}

Keywords: Longwave radiation, Pampa, clear sky, air temperature, vapor pressure. 


\section{Introdução}

A radiação de onda longa proveniente da atmosfera é uma importante componente do balanço de radiação utilizada em modelagem atmosférica. No entanto, medidas desta componente não são realizadas usualmente por estações convencionais, além disso, os instrumentos utilizados para tanto, em geral, emitem radiação em comprimentos de onda e intensidade comparáveis aos da suposta medida (Von Randow e Alvalá, 2006). Equações empíricas e analíticas são utilizadas para a estimativa da radiação de onda longa em locais onde estas medidas não são realizadas. Em geral, estas equações utilizam medidas da temperatura do ar, humidade relativa e da pressão de vapor d'água (Prata, 1996). Porém, a maioria dessas equações estima a radiação de onda longa atmosférica para dias de céu claro e foram calibradas para o Hemisfério Norte. Visando a necessidade de se obter equações de radiação de onda longa atmosférica que representem os diferentes climas terrestres, o objetivo deste trabalho é avaliar seis equações que estimam a radiação de onda longa atmosférica, e compará-las com dados experimentais obtidos num sitio experimental situado no bioma Pampa, fazendo um ajuste dos coeficientes das equações originais. Os dados experimentais de radiação analisados são do ano de 2014.

\section{Material e métodos:}

Os dados utilizados no presente trabalho foram coletados por uma torre micrometeorológica em um sítio experimental de campo nativo no bioma Pampa, localizado no município de Santa Maria-RS, pertencente a Universidade Federal de Santa Maria - UFSM (Latitude: $-29,7241^{\circ}$; Longitude: $-53,7597^{\circ}$; Altitude: $88 \mathrm{~m}$ ). Na torre micrometeorológica a radiação de onda longa é medida pelo sensor CNR 4 Net Radiometer e a umidade relativa e a temperatura pelo Termohigrômetro HMP155/Vaisala, todos a 3m de altura.
As estimativas da radiação de onda longa atmosférica incidente na superfície (Lin) foram realizadas a partir das equações de Brunt (1932), Swinbank (1963), Idso e Jackson (1969), Brutsaert (1975) e Anderson (1954), conforme a Tabela 1.

Tabela 1. Equações clássicas para a radiação de onda longa atmosférica (Lin).

\begin{tabular}{|c|c|c|}
\hline Autor & Equação* & Coeficientes \\
\hline $\begin{array}{l}\text { Brunt } \\
(1932)\end{array}$ & $\mathrm{Lin}=\mathrm{A}+\mathrm{B}\left(\mathrm{e}_{\mathrm{a}}\right)^{1 / 2} \sigma \mathrm{T}^{4}$ & $\begin{array}{l}\mathrm{A}=0.52 \\
\mathrm{~B}=0.065\end{array}$ \\
\hline $\begin{array}{l}\text { Anderson } \\
(1954)\end{array}$ & $\mathrm{Lin}=\mathrm{A}+\mathrm{B}\left(10 \mathrm{e}_{\mathrm{a}}\right)^{1 / 2} \sigma \mathrm{T}^{4}$ & $\begin{array}{l}\mathrm{A}=0.68 \\
\mathrm{~B}=0.036\end{array}$ \\
\hline $\begin{array}{l}\text { Swinbank } \\
\text { (1963) }\end{array}$ & $\mathrm{L}^{\mathrm{in}}=\left(\mathrm{AT}^{2}\right) \sigma \mathrm{T}^{4}$ & $A=9.2 \times 10^{-6}$ \\
\hline $\begin{array}{c}\text { Idso e } \\
\text { Jacson (1969) }\end{array}$ & $\begin{array}{l}\mathrm{L}^{\mathrm{in}}=(1-\mathrm{Aexp}(\mathrm{B}(273- \\
\left.\left.\mathrm{T})^{2}\right)\right) \sigma \mathrm{T}^{4}\end{array}$ & $\begin{array}{l}\mathrm{A}=0.261 \\
\mathrm{~B}=-0.000777\end{array}$ \\
\hline $\begin{array}{l}\text { Brutsaert } \\
(1975)\end{array}$ & $\mathrm{Lin}=\mathrm{A}\left(\mathrm{T} / \mathrm{e}_{\mathrm{a}}\right)^{1 / 7} \sigma \mathrm{T}^{4}$ & $\mathrm{~A}=1.24$ \\
\hline $\begin{array}{l}\text { Satterlund } \\
(1979)\end{array}$ & $\mathrm{Lin}=\mathrm{A}\left(1-\exp \left(-\mathrm{e}^{\mathrm{T} / \mathrm{B}}\right) \sigma \mathrm{T}^{4}\right.$ & $\begin{array}{l}A=1.08 \\
B=2016\end{array}$ \\
\hline $\begin{array}{l}{ }^{*} \sigma, \quad \text { const } \\
\mathrm{W} / \mathrm{m} 2 \mathrm{~K} 4) ; \mathrm{e}, \\
\text { temperatura d } \\
\text { d'água; ea, a pr }\end{array}$ & $\begin{array}{l}\text { ante de Stefan-Boltzman } \\
\text { pressão de vapor d'águ } \\
\text { ar (K); es, pressão de sat }\end{array}$ & $\begin{array}{l}(5,6697 \times 10-8 \\
(\mathrm{hPa}) ; \mathrm{T}, \mathrm{a} \\
\text { ço do vapor }\end{array}$ \\
\hline
\end{tabular}

A pressão de saturação do vapor d'água (es) foi calculada a partir da equação de Tetens (1930) e a pressão atual do vapor d'água (ea) foi calculada utilizando a es e a umidade relativa.

Como as equações avaliadas (Tabela 1) foram desenvolvidas para estimar a radiação de onda longa atmosférica apenas sob condições de céu claro, foi realizada uma caracterização de dias de céu claro com base na razão entre a irradiância solar global (Rg) e a irradiância solar no topo da atmosfera (Ro), Rg/Ro, utilizando a metodologia empregada por Ricielli (1998). Os dias considerados como dias de céu claro foram aqueles em que a razão entre a Rg e a Ro foi superior a 0,65. A radiação solar extraterrestre diária e horária foi estimada usando a relação geométrica Terra-Sol (Iqbal, 1983) e a constante solar $1367 \mathrm{~W} / \mathrm{m}^{2}$. Os dados A e B usados na Tabela 1 são dados da literatura. 


\section{Resultados}

Do período analisado (ano de 2014), 184 dias foram de céu claro. A Figura 1 mostra o ciclo diurno médio da radiação de onda longa atmosférica calculada empregando as equações clássicas (Tabela 1) e a Lin experimental (Dados Experimentais).

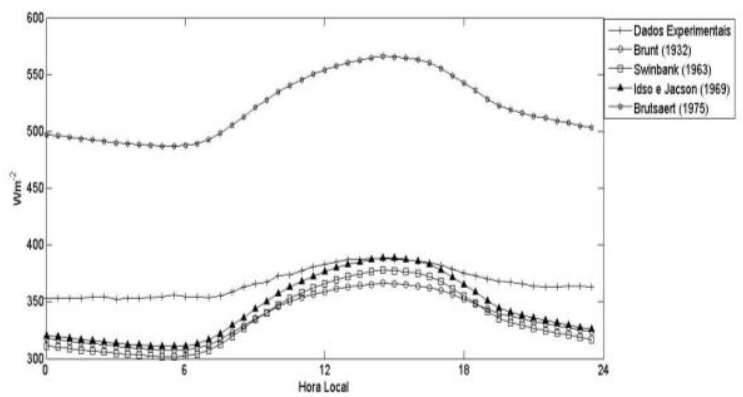

Figura 1: Ciclo diurno médio da radiação de onda longa atmosférica experimental e calculada usando as equações e parâmetros A e B da Tabela1.

As equações de Anderson (1954) e Satterlund (1979) não se ajustaram aos dados experimentais de radiação de onda longa atmosférico, pois ultrapassaram a ordem de $10^{5}$ $\mathrm{W} / \mathrm{m}^{2}$.

Observa-se que o Brunt (1932) superestimou os valores obtidos experimentalmente em mais de $150 \mathrm{~W} / \mathrm{m}^{2}$, com alto RMSE (Tabela 2) enquanto que os demais autores subestimaram tais resultados, mas com RMSE próximos. Para o horário do entre 11 e 16 h, a equação de Idso e Jacson (1969) estimou uma radiação de onda longa atmosférica de $388.4 \mathrm{~W} / \mathrm{m}^{2}$. Este valor foi o que mais se aproximou do valor experimental para este horário $\left(388.8 \mathrm{~W} / \mathrm{m}^{2}\right)$. Porém, para a noite esta equação não respondeu a magnitude do fenômeno, variando de $314.2 \mathrm{~W} / \mathrm{m}^{2}$ das 0 as $6 \mathrm{~h}$ da manhã e em torno de $331.9 \mathrm{~W} / \mathrm{m}^{2}$ das 18 as $24 \mathrm{~h}$, enquanto experimentalmente obteve-se valores de aproximadamente $350 \mathrm{~W} / \mathrm{m}^{2}$.

As equações de Swinbank (1963) e Brunt (1932) apresentaram um comportamento semelhante ao longo do ciclo diurno, com valores sempre menores que os experimentais.

Observando a discrepância entre os valores observados e estimados pelas equações clássicas calibrou-se cada uma delas para a região do Pampa utilizando o método dos mínimos quadrados para estimativa de $\mathrm{A}$ e $\mathrm{B}$ (Tabela 2). A Figura 2 mostra as novas estimativas para a Lin empregando os coeficientes A e B ajustados ao Pampa no sul do Brasil.

Tabela 2. Coeficientes A e B ajustados para o bioma Pampa no Sul do Brasil.

\begin{tabular}{l|l}
\hline \multicolumn{1}{c|}{ Autor } & \multicolumn{1}{c}{$\begin{array}{c}\text { Coeficientes } \\
\text { ajustados }\end{array}$} \\
\hline Brunt (1932) & $\mathrm{A}=146.85 \mathrm{~B}=0.125$ \\
\hline Anderson (1954) & $\mathrm{A}=1 \mathrm{~B}=0.0639$ \\
\hline Swinbank (1963) & $\mathrm{A}=1.025 \mathrm{E}^{-5}$ \\
\hline Idso e Jacson (1969) & $\mathrm{A}=0.26 \mathrm{~B}=-7.77 \mathrm{E}^{-4}$ \\
\hline Brutsaert (1975) & $\mathrm{A}=0.889279$ \\
\hline
\end{tabular}

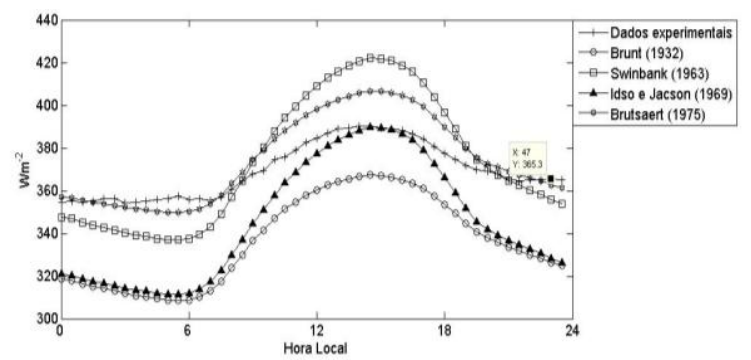

Figura 2: Ciclo diurno médio da radiação de onda longa atmosférica experimental e calculada usando as equações da Tabela1 e os parâmetros ajustados A e B da Tabela 2.

Tabela 3: RMSE diário médio para o período para as estimativas de Lin para a região do PAMPA

\begin{tabular}{c|c|c}
\hline Modelo & $\begin{array}{c}\text { Usando A e } \\
\text { B da Tabela 1 }\end{array}$ & $\begin{array}{c}\text { Usando A e B } \\
\text { estimado pelo } \\
\text { presente trabalho }\end{array}$ \\
\hline $\begin{array}{c}\text { Brunt } \\
(1932)\end{array}$ & 33.27 & 9.86 \\
\hline $\begin{array}{c}\text { Swinbank } \\
(1963)\end{array}$ & 35.76 & 9.91 \\
\hline $\begin{array}{c}\text { Idso e } \\
\text { Jacson (1969) }\end{array}$ & 28.51 & 9.91 \\
\hline $\begin{array}{c}\text { Brutsaert } \\
(1975)\end{array}$ & 154.91 & 9.37 \\
\hline
\end{tabular}


A estimativa da radiação de onda longa atmosférica utilizando os parâmetros A e B ajustados mostra que a equação de Brutsaert (1975) foi a que mais se aproximou dos valores experimentais, com menor RMSE, obtendo o valor de $406.7 \mathrm{~W} / \mathrm{m}^{2}$ para o período entre meio dia e as 16hs, sendo que os valores experimentais para este mesmo horário são da ordem de $380 \mathrm{~W} / \mathrm{m}^{2}$; o período das 0 as $6 \mathrm{~h}$ os valores ficaram próximos aos $360 \mathrm{~W} / \mathrm{m}^{2}$ tanto experimental como o valor obtidos pela equação de Brutsaert (1975). No período noturno, das 19 a $24 \mathrm{~h}$, observa-se valores muito próximos de $350 \mathrm{~W} / \mathrm{m}^{2}$, correspondendo aos valores experimentais.

A equação de Swinbank (1963) superestimou o valor observado em cerca de 30 $\mathrm{W} / \mathrm{m}^{2}$ e ficou na média de $421.7 \mathrm{~W} / \mathrm{m}^{2}$ entre 10 e $16 \mathrm{~h}$. Das 0 as $6 \mathrm{~h}$ tivemos este modelo subestimando o valor experimental assumindo valores da ordem de $367 \mathrm{~W} / \mathrm{m}^{2}$ enquanto que o valor experimental foi de $356 \mathrm{~W} / \mathrm{m}^{2}$, levando em conta que o valor do RMSE (9.91) não apresentou muita variação com os índices da literatura.

Equação de Idso e Jacson (1969) entre 14 e 16h apresentou valores muito próximos do experimental e, após as $18 \mathrm{~h}$ os valores experimentais são subestimado, com diferença de quase $30 \mathrm{~W} / \mathrm{m}^{2}$. A equação de Brunt (1932) subestimou a Lin experimental para todo o período, tento quase $40 \mathrm{~W} / \mathrm{m}^{2}$ de diferença no horário próximo as $16 \mathrm{~h}$, apresentando valores de RMSE próximo dos maiores, quando do uso dos coeficientes calibrados. Porém por ter subestimado em ambas os casos, ou seja, com coeficientes da literatura e com coeficientes estimados, esta não seria uma boa aproximação para descrever a radiação de onda longa atmosférica sobre o Pampa.

\section{Conclusões}

As equações que levam em consideração a pressão de vapor d'água e a temperatura do ar tiveram melhor desempenho na estimativa da radiação de onda longa atmosférica que as equações que utilizam apenas a temperatura do ar, como é o caso das equações de Swinbank (1963) e Idso e Jackson (1969). A equação de Brutsaert (1975) apresentou o melhor desempenho, obtendo os maiores índices de concordância, e sendo, portanto, a equação mais indicadas para a estimativa da radiação de onda longa atmosférica na região do Pampa no Sul do Brasil.

Por sua vez, torna-se evidente também, a necessidade de novas parametrizações para estimar a radiação de onda longa atmosférica que possam ser utilizadas para todas as condições de cobertura do céu.

\section{Agradecimentos}

\section{CAPES, CNPQ}

\section{Referências}

VON RANDOW, R. C. S.; ALVALÁ, R. C. S. Estimativa da Radiação de Onda Longa Atmosférica no Pantanal Sul Mato-Grossense Durante os Períodos Secos de 1999 e 2000. Revista Brasileira de Meteorologia. v. 21, n. 3b, p. 398-412, 2006.

PRATA, A .J. A new long-wave formula for estimating downward clear-sky radiation at the surface. Quarterly Journal Resource Meteorological Society, v. 122, p. 1127-1151, 1996.

TETENS, O. Uber cinige meterorologische Begriffe. Z. Geophys., n.6, p. 297-309, 1930.

BRUNT, D. Notes on radiation in the atmosphere. Quarterly Journal Resource Meteorological Society, v. 58, p. 389-418, 1932.

BRUTSAERT, W. On a derivable formula for long-wave radiation from clear skies. Water Resources Research, v. 11, n. 5, p. 742-744, Oct. 1975. 
GALVÃO, J. A. C.; FISCH, G. Balanço de radiação em área de pastagem na Amazônia. Revista Brasileira de Agrometeorologia. v. 8, n. 1, p. 1-10, 2000.

IDSO, S. B.; JACKSON R. D. Thermal Radiation from the Atmosphere. Journal Geophysics Research, v. 74, n. 23, p. 5397-5403, 1969.

IDSO, S. B. A set of equations for full spectrum and 8 to $14 \mu \mathrm{m}$ and 10.5 to $12.5 \mu \mathrm{m}$ thermal radiation from cloudless skies. Water Resources Research, v. 17, n. 2, p. 295-304, 1981.
IQBAL, M., 1983. An Introduction to Solar Radiation. Academic Press, Toronto.

RICIERI, R.P. Modelos de estimativa e avaliação dos métodos de medida da radiação solar difusa, UNESP, Botucatu, 1998. 81 p. Tese (Doutorado) - Programa de Pós Graduação em Energia na Agricultura - Universidade Estadual Paulista, Faculdade de Ciências Agronômicas, Botucatu, 1998. 